\title{
ON THE HOMOTOPY TYPE OF COMPACT TOPOLOGICAL MANIFOLDS
}

\author{
BY L. C. SIEBENMANN ${ }^{1}$
}

Communicated by W. Browder, December 1, 1967

1. In 1964, C. T. C. Wall introduced a new species of torsion [11], [12]. It is an invariant of ordinary homotopy type, defined for any path connected Hausdorff space $X$ that is a retract-up-to-homotopy of a finite complex. For example, $X$ can be a compact ANR (cf. $[1, p$. 106]). This torsion, denoted $\sigma(X)$, lies in $\tilde{K}_{0} Z\left[\pi_{1} X\right]$, the projective class group $^{2}$ of the integral group-ring of $\pi_{1}(X)$. Its vanishing is a necessary and sufficient condition that $X$ have the homotopy type of a finite complex. Now it has been conjectured by Borsuk since 1954 $[16$, p. 203] $[1$, p. 218] that every compact ANR $X$ has the homotopy type of a finite complex, i.e. has $\sigma(X)=0$. For a compact topological manifold $X$ this conjecture is of particular interest, because if $\sigma(X) \neq 0, X$ can certainly not be triangulated by a finite complex as the long-standing 'triangulation conjecture' asserts.

Let $X$ be a connected compact topological manifold possibly with boundary. Let $L=L_{1} \cup L_{2}$ be a connected simplicial (or CW-) complex expressed as the union of two subcomplexes, ${ }^{3}$ and let $f: X \rightarrow L$ be any continuous map.

THEOREM I. $f_{*} \sigma(X)$ lies in the subgroup of $\widetilde{K}_{0} Z\left[\pi_{1} L\right]$ generated by the images under inclusion of $\tilde{K}_{0} Z\left[\pi_{1} L_{1}\right]$ and $\tilde{K}_{0} Z\left[\pi_{1} L_{2}\right]$.

With a little homotopy theory this gives

Corollary II. If $X$ admits a map to a 3-complex inducing an isomorphism of fundamental groups, then $\sigma(X)=0$, i.e. $X$ has the homotopy type of a finite complex.

Corollary III. If $X$ has dimension $\leqq 4, X$ has the homotopy type of a finite complex.

If the boundary $b X$ is nonempty, Corollary III is a special case of Corollary II. If $X$ is closed, remove a small open disc and apply Corollary II with [11].

1 The author gratefully acknowledges the hospitality of the University of Liverpool where he was visiting July to October 1967 when this work was done.

2 If $\pi_{1} X=1$ one has $\widetilde{K}_{0} Z\left[\pi_{1} X\right]=0$ and so $\sigma(X)$ is then zero. 'Torsion' conveys the idea of twisting of structure along noncontractible loops.

3 The subcomplexes need not be connected. If $A$ is a nonconnected space, $\tilde{K}_{0} Z\left[\pi_{1} A\right]$ is by definition $\oplus\left\{\tilde{K}_{0} Z\left[\pi_{1} A_{\alpha}\right] \mid A_{\alpha}\right.$ an arc-component of $\left.A\right\}$. 
Theorem I can be used to add to a result of A. Casson (unpublished) as follows:

COROLLARY IV. If $h: M_{1} \rightarrow M_{2}$ is a homeomorphism of compact connected PL manifolds, and if $M_{1}$ admits a map to a 3-complex $K$ inducing an isomorphism of fundamental groups, then $h$ is a simple homotopy equivalence.

The addition is slight since Casson proves this if $K$ is a 2-complex or a 3-complex with abelian fundamental group. What is more, Casson's result applies equally to homeomorphisms of finite simplicial complexes. Again, Corollary IV should be contrasted with

Proposition (CF. ARGUMENT IN [2] ${ }^{4}$ ). If simple homotopy type of compact PL manifolds is a topological invariant (i.e. if Corollary IV holds without mention of $K$ ), then every compact topological manifold has the homotopy type of a finite complex.

The starting point for Theorem I was a basic observation ${ }^{5}$ of A. Casson who communicated to me the Hsiang-Farrell examples of $h$ cobordisms that are not topologically a product [14]. Casson pointed out that numerous simpler examples exist as follows: Let there be given a closed connected smooth manifold $V^{n}$ of dimension $n \geqq 6$ and a splitting $V=X_{1} \cup X_{2}$ of $V$ in to two smooth compact $n$-submanifolds intersecting in a common boundary $b X_{1}=b X_{2}=X_{0}$. Suppose that

$$
0=\mathrm{Wh} \pi_{1} X_{1}=\mathrm{Wh} \pi_{1} X_{2}=K_{0} Z\left[\pi_{1} X_{0}\right] \text {, but } \mathrm{Wh} \pi_{1} V \neq 0 \text {. }
$$

The simplest examples have $\pi_{1} V=Z_{5}$ and the three other $\pi_{1}$ 's equal $Z$ or 1 . Other examples include any $V$ that is a boundary of a regular neighborhood of a finite connected 2-complex in euclidean space. The assertion is that no $h$-cobordism $c=\left(W ; V, V^{\prime}\right)$ with nonzero Whitehead torsion $\tau(c) \in \mathrm{Wh} \pi_{1} V$ (from $V \hookrightarrow W$ ) can be topologically a product. For suppose a homeomorphism $h: V \times I \rightarrow W$ does exist (with $h \mid V \times 0$ inclusion for convenience). If $N \cong X_{0} \times R^{1}$ is a bicollar neighborhood of $X_{0}$, the 2-ended smooth manifold $h(N \times I) \subset W$ splits as smooth product $h(N \times I)=M \times R^{1}$ with $M \times 0 \cap V=X_{0}$, because the only obstruction to this lies in $\tilde{K}_{0} Z\left[\pi_{1} X_{0}\right]=0$-see [5] and $\$ 2$ below. Then $W$ is split by $M \times 0$ as a union of two $h$-cobordisms with boundary $c_{1}=\left(W_{1} ; X_{1}, X_{1}^{\prime}\right)$ and $c_{2}=\left(W_{2} ; X_{2}, X_{2}^{\prime}\right)$ with $W_{1} \cap W_{2}$

4 In [2], which is unpublished, Gersten assumes the Hauptvermutung for PL manifolds and deduces that twice Wall's obstruction is zero for each closed oriented topological manifold.

- Added in proof. E. H. Connell makes a somewhat similar observation in Bull. Amer. Math. Soc. 74 (1968), 176-178. 
$=M \times 0$. A sum-theorem for Whitehead torsions [5, Chapter VI] now shows that $\tau(c)=0$, since $\tau\left(c_{1}\right), \tau\left(c_{2}\right)$ (being in zero groups!) are both zero.

The author is much indebted to A. Casson for passing on the above observation and also for helpful conversations about this work.

The steps in proving Theorem I are as follows:

(1) By geometric manipulations reduce I to a parallel assertion $I^{\prime}$ concerning the invariant of a 'pseudo-product' (defined precisely in \$2-A), which happens to be homeomorphic to the product of a compact PL manifold with the line.

(2) Prove I' by splitting the pseudo-product much in the way Casson's observation suggests. Infinite surgery is involved here-the worst of which is evaded by the device of 'gluing' explained in [8]. The sum-theorem for Wall's invariant [5], [12] completes the proof. Detailed proofs will be published elsewhere.

2. Applications. We will consistently discuss smooth paracompact manifolds, but similar results with essentially the same proofs hold for PL (= piecewise linear, paracompact) manifolds.

(A) Pseudo-products. If $M^{n}$ is a connected smooth $n$-manifold such that for some closed smooth connected manifold $S, M \times S$ is a smooth product $N \times R^{1}$ of a compact manifold $N$ with the line $R^{1}$, then we call $M$ a pseudo-product. In [4], [5], [6], [9] can be found necessary and sufficient homotopy theoretic conditions that $M$ be a pseudoproduct. For example, if $b M=\varnothing$, it is necessary and sufficient that there exist a finite complex $K$ and proper maps $r: K \times R^{1} \rightarrow M$ and $i: M \rightarrow K \times R^{1}$ such that $r i$ is proper homotopic to $1 \mid M$ and $r_{*}: \pi_{1} K$ $\times R^{1} \rightarrow \pi_{1} M$ is an isomorphism. If $b M \neq \varnothing$, one must add the similar condition on $b M$. Note that $M$ necessarily has two ends, which we denote $\epsilon_{-}, \epsilon_{+}$.

If $M^{n}$ is a pseudo-product, then $M$ may or may not be a smooth product with $R^{1}$. In fact there remains an obstruction, ${ }^{6} \sigma\left(\epsilon_{+}\right)$ $\in \widetilde{K}_{0} Z\left[\pi_{1} M\right]$ and arbitrary obstructions occur for $n \geqq 5$. If $n \geqq 6$ and $b M$ is already a smooth product with $R^{1}$, then by [5] the product structure can be extended over $M$ if and only if $\sigma\left(\epsilon_{+}\right)=0$. The obstruction $\sigma\left(\epsilon_{+}\right)$comes from Wall's obstruction to finiteness $\sigma(V)$ for any closed neighborhood $V$ of $\epsilon_{+}$that is a smooth submanifold (with boundary). ${ }^{7}$ The geometric characterization of pseudo-products mentioned first, exists because by a product theorem for Wall's obstruc-

- $\sigma\left(\epsilon_{-}\right)$would be $(-1)^{n+1} \sigma\left(\epsilon_{+}\right)^{*}$ for an involution* of $\tilde{K}_{0} Z\left[\pi_{2} M\right]$ determined by the Stiefel-Whitney class $w_{1}(M)$.

$7 V$ must also be disjoint from some neighborhood of $\epsilon_{-}$. 
tion $\sigma\left(\epsilon_{+} \times S\right)=\chi(S) j * \sigma\left(\epsilon_{+}\right) \in K_{0} Z\left[\pi_{1}(M \times S)\right]$, where $\chi(S)$ is the Euler characteristic and $j$ is inclusion $M \hookrightarrow M \times S$.

Suppose now that $M$ is a smooth connected pseudo-product such that there exists a continuous map of $M$ to a 3-complex inducing an isomorphism of fundamental group. By arguments based on Corollary II one finds:

(i) If $\sigma(M) \neq 0, M$ cannot be homeomorphic to $X \times R^{1}$ for $X$ any topological space whatever.

(ii) If $M^{\prime}$ is $M$ with a new smooth or PL structure, then $\sigma\left(\epsilon_{+}^{\prime}\right)=\sigma\left(\epsilon_{+}\right)$. In other words $\sigma\left(\epsilon_{+}\right)$is a topological invariant.

Part (i) is remarkable, because, if $n \geqq 6$ (or $n \geqq 5$ and $b M=\varnothing$ ), $M$ can be smoothly isotoped into any prescribed neighborhood of $\epsilon_{+}$ through embeddings into $M$ that all fix a smaller (=more remote) neighborhood of $\epsilon_{+}$[6]. To illuminate (ii) we remark that while $\sigma\left(\epsilon_{+}\right)$is obviously a diffeomorphism invariant, it is not a homotopy or even a proper homotopy invariant. It is best regarded as an invariant of infinite simple homotopy type. I shall discuss this notion elsewhere in connection with an extension of the $s$-cobordism theorem to noncompact manifolds-cf. [7].

The foregoing can easily be formulated for the tame ends of [5] rather than for pseudo-products. The basic fact is that every smooth tame end has a neighborhood that is a pseudo-product, and any two such are diffeomorphic fixing a smaller neighborhood [6]. The present formulation is designed to emphasize parallelism with the theory of $h$-cobordisms.

(B) Distinguishing knots. J. Sondow [15] studied the class of smooth knots $\mathscr{K}=\left(S^{n}, K^{n-2}\right)$ of the ordinary sphere $S^{n-2}$ in $S^{n}$ such that $\left(S^{n}, K^{n-2}\right) \times R^{1}$ is diffeomorphic to $\left(S^{n}, K_{0}^{n-2}\right) \times R^{1}$ for a fixed knot $K_{0}=\left(S^{n}, K_{0}^{n-2}\right)$. In case $\varkappa_{0}$ is a repeatedly 5-twist-spun trefoil [13] and $n$ is odd $\geqq 5$, he found an infinite sequence $\mathfrak{K}_{1}, \mathfrak{K}_{2}$, $\mathfrak{K}_{3}, \ldots$ distinguished by Reidemeister torsions. They are in fact all topologically distinct although their suspensions are clearly all homeomorphic. For Corollary IV (even in Casson's version) applies to the ( $C^{1}$-triangulated) 10 -fold branched cyclic coverings of the $K_{i}$, and the result readily follows with the help of the torsions. Compare [10].

For another example we begin with a knot in $S^{3}$ whose 2-fold cyclic branched covering space in a lens space [3, Satz 6] with fundamental group cyclic of prime order 229. Let the knot $\left(S^{n}, K_{0}^{n-2}\right)$, $n$ odd $\geqq 5$, be obtained by repeatedly 2 -twist-spinning the above knot. Then by [13] $S^{n}-K_{0}$ is smoothly fibered over the circle so that the closure of each fiber is a compact manifold with boundary $K_{0}$. Using a class group invariant we construct a smooth knot $\left(S^{n}, K^{n-2}\right)$ such 
that $\left(S^{n}, K_{0}\right) \times R^{1}$ is diffeomorphic to $\left(S^{n}, K\right) \times R^{1}$ and yet $S^{n}-K$ cannot fiber topologically over the circle with fiber the interior of a compact topological manifold. If it did, statement (i) in part (A) of this section would be contradicted.

Added in proof. [16] was called to our attention by C. B. de Lyra, who independently constructed $\sigma(X)$ in important cases (see [12]).

\section{REFERENCES}

1. K. Borsuk, The theory of retracts, Polish Scientific Publishers, Warsaw, 1967.

2. S. Gersten, A relation between two conjectures in topology, preprint, Oxford Mathematical Institute, 1965.

3. H. Schubert, Knoten mit zwei Briicken, Math. Z. 65 (1950), 133-170.

4. L. C. Siebenmann, Finding a boundary for an open manifold, Notices Amer. Math. Soc. 12 (1965), 337.

5. - Doctoral dissertation, Princeton University, 1965, Dissertation Abstracts 27 (June 1966), 2044-2045 (No. 66-5012), University Microfilms Ltd., 300 N. Zeeb Rd., Ann Arbor, Mich.

6. - The structure of tame ends, Notices Amer. Math. Soc. 13 (1966), 862.

7. — Notices Amer. Math. Soc. 14 (1967), 852.

8. - (to appear).

9. - - (version of [5] to appear).

10. L. C. Siebenmann and J. Sondow, Some homemorphic sphere pairs that are combinatorially distinct, Comment. Math. Helv. 41 (1966-1967), 261-272.

11. C. T. C. Wall, Finiteness conditions for CW-complexes, Ann. of Math. 81 (1965), 56-69.

12. - Finiteness conditions for CW-complexes. II, Proc. Roy. Soc. Ser. A 295 (1966), 129-139.

13. E. C. Zeeman, Twisting spun knots, Trans. Amer. Math. Soc. 115 (1965), 471495.

14. F. T. Farrell and W. C. Hsiang, H-cobordant manifolds are not necessarily homeomorphic, Bull. Amer. Math. Soc. 73 (1967), 741-744. 1965.

15. J. Sondow, Doctoral dissertation, Princeton University, Princeton, N. J.,

16. K. Borsuk, Sur l'élimination de phenomènes paradoxaux en topologie générale, Proc. Int. Congress of Mathematicians, Amsterdam, 1954, Vol. I, pp. 197-208.

Faculte des Sciences

91 Orsay, France 